\title{
https://doi.org/10.46813/2021-136-103 \\ TRANSITION RADIATION OF A RELATIVISTIC ELECTRON BUNCH ON A SEMI-INFINITE METAL CYLINDER
}

\author{
V.A. Balakirev, I.N. Onishchenko \\ National Science Center "Kharkov Institute of Physics and Technology”, Kharkiv, Ukraine \\ E-mail: onish@kipt.kharkov.ua
}

Transition radiation of a relativistic electron bunch, which arises when it collides with the end face of a semiinfinite ideally conducting cylinder, is considered. An electron bunch moves along the axis of a semi-infinite cylinder. Expressions for the field strength of electromagnetic radiation in the wave zone are obtained. The influence of the guiding properties of an ideally conducting cylinder on the directional diagram of the transition radiation is investigated.

PACS: 41.75.Lx, 41.85.Ja, 41.69.Bq

\section{INTRODUCTION}

The effects of transition and diffraction radiation of relativistic electron bunches [ 1 - 4] can underlie the processes of excitation of pulsed electromagnetic radiation in a wide frequency range [5 - 10]. In [10] the process of excitation of an infinite ideally conducting cylinder by a relativistic electron bunch was investigated. Thereat the situation was considered when an electron bunch collides with a cylinder perpendicular to its cylindrical surface. Below we will consider a semi-infinite ideally conducting cylinder, which is excited by a relativistic electron bunch when it collides with the end face of the cylinder. It is in this way the ultra-wideband antennas were excited by a relativistic electron beam [7 - 9]. Expressions for the intensity of electromagnetic radiation in the wave zone are obtained. The directional diagram of transition radiation and, first of all, the influence of the guiding properties of a cylindrical conductor on its formation is investigated.

\section{STATEMENT OF THE PROBLEM. BASIC EQUATIONS}

We consider a semi-infinite ideally conducting circular cylinder of radius $a$. A relativistic electron bunch moves in vacuum along the cylinder axis and collides with its face end. For simplicity, we will consider an infinitely thin tubular electron bunch with a current density

$$
\vec{j}=-\vec{e}_{z} Q \frac{\delta\left(r-r_{0}\right)}{2 \pi r} \frac{1}{t_{b}} T\left(\frac{t-z / v_{0}}{t_{b}}\right),
$$

where $\vec{e}_{z}$ is the unit vector in the longitudinal direction, $Q$ is the charge of the bunch, $r_{0}$ is the radius of the bunch, $r_{0}<a, v_{0}, t_{b}$ are velocity and duration of the bunch, $\delta\left(r-r_{0}\right)$ is the delta function. The dimensionless function $T(\tau)$ describes the longitudinal density profile of the bunch and satisfies the normalization condition

$$
\int_{-\infty}^{\infty} T(\tau) d \tau=1
$$

where $T(\tau)=T(-\tau)$ is symmetrical function.

Let us represent the excited electromagnetic field and the current density in the form of Fourier integrals over frequencies. So, for example, for a magnetic field we have

$$
\vec{H}(r, z, t)=\int_{-\infty}^{\infty} \vec{H}_{\omega}(r, z) e^{-i \omega t} d \omega,
$$

where

$$
\vec{H}_{\omega}(r, z)=\frac{1}{2 \pi} \int_{-\infty}^{\infty} \vec{H}(r, z, t) e^{i \omega t} d t
$$

is Fourier amplitude of the magnetic field.

The system of Maxwell's equations for the components of the Fourier amplitudes of the electromagnetic field has the form

$$
\begin{gathered}
\frac{\partial E_{\omega r}}{\partial z}-\frac{\partial E_{\omega z}}{\partial r}=i k_{0} H_{\omega \varphi} \\
\frac{1}{r} \frac{\partial}{\partial r} r \frac{\partial}{\partial r} H_{\omega \varphi}+i k_{0} E_{\omega z}=-\frac{2 Q}{c t_{b}} \frac{\delta\left(r-r_{0}\right)}{r} T_{\omega} e^{i k_{l} z} \\
\frac{\partial H_{\omega \varphi}}{\partial z}=-i k_{0} E_{\omega r},
\end{gathered}
$$

where $k_{0}=\omega / c, k_{l}=\omega / v_{0}$,

$$
T_{\omega}=\frac{1}{2 \pi} \int_{-\infty}^{\infty} T\left(\frac{t_{0}}{t_{b}}\right) e^{i \omega t_{0}} d t_{0} .
$$

Let's divide the space into three regions:

$$
\begin{gathered}
I-r_{0} \geq r \geq 0, \quad z<0 ; \\
I I-a \geq r \geq r_{0}, \quad z<0 ; \\
I I I-\infty>r \geq a, \infty>z>-\infty .
\end{gathered}
$$

The problem is reduced to solving the inhomogeneous system of Maxwell's equations (3) with the following boundary conditions. On the current surface $r=r_{0}$, the longitudinal component of the electric field is continuous, and the magnetic field experiences a leap

$$
\begin{aligned}
& r=r_{0}, \quad E_{\omega z}^{I I}(z<0)=E_{\omega z}^{I}(z<0), \\
& H_{\omega \varphi}^{I I}(z<0)-H_{\omega \varphi}^{I}(z<0)=-\frac{2 Q}{c t_{b} r_{0}} T_{\omega} e^{i k_{l} z} .
\end{aligned}
$$

On the boundary $r=a$ in the left half-space $z<0$, the tangential components of the electromagnetic field are continuous

$$
\begin{gathered}
r=a, \quad E_{\omega z}^{I I I}(z<0)=E_{\omega z}^{I I}(z<0), \\
H_{\omega \varphi}^{I I I}(z<0)=H_{\omega \varphi}^{I I}(z<0) .
\end{gathered}
$$

In the right half-space $z>0$ on the surface of a perfectly conductive cylinder, the longitudinal component of the electric field vanishes

$$
r=a, \quad E_{\omega z}^{I I I}(z>0)=0 .
$$

And, finally, at the end of a semi-infinite cylinder, the radial component of the electric field also vanishes

$$
a>r>0, E_{\omega r}^{I}(z=0, r)=E_{\omega r}^{I I}(z=0, r)=0 .
$$




\section{METHOD OF SOLUTION. MAIN RESULTS}

In the system under consideration, the electromagnetic field disappears at infinity, so the electromagnetic field can be sought in the form of a superposition of waves propagating in the longitudinal direction

$$
\begin{gathered}
E_{\omega z}^{I}(r, z)=\int_{-\infty}^{\infty} e_{1}(k) J_{0}(v r) e^{i k z} d k \\
H_{\omega \varphi}^{I}(r, z)=-i k_{0} \int_{-\infty}^{\infty} e_{1}(k) v^{-1} J_{1}(v r) e^{i k z} d k \\
E_{\omega z}^{I I}(r, z)=\int_{-\infty}^{\infty}\left[e_{2}(k) J_{0}(v r)+e_{3}(k) H_{0}^{(1)}(v r)\right] e^{i k z} d k \\
H_{\omega \varphi}^{I I}(r, z)=-i k_{0} \int_{-\infty}^{\infty} v^{-1}\left[e_{2} J_{1}(v r)+e_{3} H_{1}^{(1)}(v r)\right] e^{i k z} d k \\
E_{\omega z}^{I I I}(r, z)=\int_{-\infty}^{\infty} e_{4}(k) H_{0}^{(1)}(v r) e^{i k z} d k \\
H_{\omega \varphi}^{I I I}(r, z)=-i k_{0} \int_{-\infty}^{\infty} e_{4}(k) v^{-1} H_{1}^{(1)}(v r) e^{i k z} d k
\end{gathered}
$$

$v=\sqrt{k_{0}^{2}-k^{2}}, e_{1}(k) \ldots e_{4}(k)$ are the sought amplitudes of the fields in the corresponding regions, $J_{n}(v r), H_{n}^{(1)}(v r)$ are the Bessel and Hankel functions, $n=0.1$.

Substituting fields (8) into boundary conditions (4) (6), we obtain a system of paired integral equations, which, using the Wiener-Paley-Rappoport lemma, can be reduced to the following system of functional equations

$$
\begin{gathered}
{\left[e_{1}(k)-e_{2}(k)\right] J_{0}\left(v r_{0}\right)-e_{3}(k) H_{0}^{(1)}\left(v r_{0}\right)=\varphi^{-}(k), \quad \text { (9a) }} \\
-i \frac{k_{0}}{v}\left\{\left[e_{1}(k)-e_{2}(k)\right] J_{1}\left(v r_{0}\right)-e_{3}(k) H_{1}^{(1)}\left(v r_{0}\right)\right\}=\psi^{-}(k)- \\
-\frac{2 Q}{c t_{b} r_{0}} T_{\omega} \frac{1}{2 \pi i\left(k-k_{l}\right)}, \\
e_{2}(k) J_{0}(v a)+\left[e_{3}(k)-e_{4}(k)\right] H_{0}^{(1)}\left(v r_{0}\right)=\theta^{-}(k), \quad(9 \mathrm{c}) \\
-i \frac{k_{0}}{v}\left\{e_{2}(k) J_{1}(v a)+\left[e_{3}(k)-e_{4}(k)\right] H_{1}^{(1)}\left(v r_{0}\right)\right\}=\lambda^{-}(k), \\
e_{4}(k) H_{0}^{(1)}\left(v r_{0}\right)=\kappa^{+}(k),
\end{gathered}
$$

where $\varphi^{-}(k), \psi^{-}(k), \theta^{-}(k), \lambda^{-}(k)$ are analytic functions in the lower half-plane of the complex variable $k$, and $\kappa^{+}(k)$ is the analitic function in the upper one, $\operatorname{Im} k_{l}<0$.

From the boundary condition at the face end of the cylinder (7) it follows that the following amplitudes are even

$$
e_{\alpha}(k)=e_{\alpha}(-k), \alpha=1,2,3 .
$$

It can be shown that the system of functional equations (9), taking into account relations (10), is equivalent to the system of Hilbert boundary value problems on the real axis $\operatorname{Im} k=0$

$$
\begin{gathered}
\varphi^{+}(k)-\varphi^{-}(k)=0 \\
\psi^{+}(k)-\psi^{-}(k)=-\frac{2 Q}{c t_{b} r_{0}} T_{\omega} \frac{k}{\pi i\left(k^{2}-k_{l}^{2}\right)}, \\
\theta^{+}(k)-\theta^{-}(k)=\kappa^{+}(k)-\kappa^{-}(k), \\
\lambda^{+}(k)-\lambda^{-}(k)=-H(k)\left[\kappa^{+}(k)-\kappa^{-}(k)\right] \\
i \frac{k_{0}}{v} J_{1}\left(v r_{0}\right)\left[\varphi^{+}(k)+\varphi^{-}(k)\right]+J_{0}\left(v r_{0}\right)\left[\psi^{+}(k)+\psi^{-}(k)\right]+
\end{gathered}
$$

$$
\begin{gathered}
+\frac{a}{r_{0}}\left\{i \frac{k_{0}}{v} J_{1}(v a)\left[\theta^{+}(k)+\theta^{-}(k)\right]+J_{0}(v a)\left[\lambda^{+}(k)+\lambda^{-}(k)\right]\right\} \\
=\frac{2 k_{0}}{\pi v^{2} r_{0}} \frac{\kappa^{+}(k)-\kappa^{+}(k)}{H_{0}^{(1)}(v a)}+\frac{2 Q}{c t_{b} r_{0}} T_{\omega} J_{0}\left(v r_{0}\right) \frac{k_{l}}{\pi i\left(k^{2}-k_{l}^{2}\right)},
\end{gathered}
$$$$
\text { where } \quad \theta^{+}(k)=\theta^{-}(-k), \lambda^{+}(k)=\lambda^{-}(-k) \quad \text { etc, }
$$$$
H(k)=\frac{i k_{0}}{v} \frac{H_{1}^{(1)}(v a)}{H_{0}^{(1)}(v a)} .
$$

Solutions of the boundary problems (11a), (11b) are found trivially

$$
\begin{gathered}
\varphi^{+}(k)=\varphi^{-}(k) \equiv 0, \\
\psi^{+}(k)=-\frac{2 Q}{c t_{b} r_{0}} T_{\omega} \frac{1}{2 \pi i\left(k-k_{l}\right)}, \\
\psi^{-}(k)=-\frac{2 Q}{c t_{b} r_{0}} T_{\omega} \frac{1}{2 \pi i\left(k+k_{l}\right)} .
\end{gathered}
$$

Using the Sokhotsky formulas [11], the system of boundary problems (12) can reduce to the following singular equation for the function $\kappa=\kappa^{+}-\kappa^{-}$

$$
\begin{array}{r}
2 I(k) \hat{L} \kappa(k)-\hat{L} H(k) \kappa(k)-H(k) \hat{L} \kappa(k)= \\
=\frac{4 Q}{c t_{b} a} T_{\omega} \frac{J_{0}\left(v r_{0}\right)}{J_{0}(v a)} \frac{k_{l}}{\pi i\left(k^{2}-k_{l}^{2}\right)},
\end{array}
$$

where

$$
I(k)=\frac{i k_{0}}{v} \frac{J_{1}(v a)}{J_{0}(v a)}, \hat{L} f(k)=\frac{1}{\pi i} \int_{-\infty}^{\infty} \frac{f\left(k^{\prime}\right)}{k^{\prime}-k} d k^{\prime} .
$$

Integral (14) should be understood in the sense of the principal value. It is not possible to find a general solution to this integral equation. However, in the limiting case of a conducting cylinder of small radius $k_{0} a<<1$, the solution can be found approximately. In this limiting case, the term in the integral equation (13), which proportional $I(k) \sim k_{0} a$ can be neglected. Then the integral equation is simplified and takes the form

$$
\hat{L} H(k) \kappa(k)+H(k) \hat{L} \kappa(k)=-\frac{4 Q}{c t_{b} a} T_{\omega} \frac{k_{l}}{\pi i\left(k^{2}-k_{l}^{2}\right)} .
$$

After replacement

$$
U(k)=H(k) \kappa(k)
$$

the simplified integral equation (15), in turn, is reduced to the boundary value problem [12]

$$
U^{-}=-H \kappa^{+}=-\frac{2 Q}{c t_{b} a} T_{\omega} \frac{k_{l}}{\pi i\left(k^{2}-k_{l}^{2}\right)},
$$

the solution of which can be found explicitly

$$
\begin{gathered}
\kappa^{+}(k)=-\frac{4 Q}{c t_{b} a} T_{\omega} \frac{k_{l}}{k_{0}} \frac{X^{+}(k) \sqrt{k_{0}+k}}{(2 \pi i)^{2}} \times \\
\times \int_{C} \frac{\sqrt{k_{0}-k^{\prime}}}{X^{-}\left(k^{\prime}\right)\left(k^{\prime 2}-k_{l}^{2}\right)\left(k^{\prime}-k\right)} d k^{\prime},
\end{gathered}
$$

where $X^{ \pm}(k)$ is solution of homogeneous conjugation problem

$$
X^{+}(k)=G(k) X^{-}(k), \quad G(k)=\frac{H_{0}^{(1)}(v a)}{i H_{1}^{(1)}(v a)} .
$$

The solution to this boundary value problem has the form [11]

$$
X^{-}(k)=\exp \frac{1}{2 \pi i} \int_{C} \frac{\ln G\left(k^{\prime}\right)}{k^{\prime}-k} d k^{\prime}, X^{+}(k)=\frac{1}{X^{-}(-k)} .
$$


The contour $C$ runs along the real axis and bypasses the singularity $k^{\prime}=k$ from below. The integral included in (16) is easily calculated by closing the integration contour into the lower half-plane. As a result, we obtain

$$
\kappa^{+}(k)=\frac{2 Q}{c t_{b} a k_{0}} T_{\omega} \frac{\sqrt{\left(k_{0}+k\right)\left(k_{0}-k_{l}\right.}}{2 \pi i\left(k_{l}-k\right)} \frac{X^{+}(k)}{X^{-}\left(k_{l}\right)} .
$$

Relation (9d), taking into account the obtained expression for the function $\kappa^{+}(k)$, makes it possible to determine the amplitude $e_{4}(k)$ for the region $r>a$ and, accordingly, the expression for the magnetic field in this region

$$
\begin{aligned}
H_{\omega \varphi}^{I I I}=-\frac{2 Q T_{\omega}}{c t_{b} a} \frac{\sqrt{k_{0}-k_{l}}}{2 \pi} & X^{-}\left(k_{l}\right) \\
& \quad \times \int_{-\infty}^{\infty} \frac{X^{+}(k) H_{1}^{(1)}(v r) e^{i k z}}{\sqrt{k_{0}-k}\left(k_{l}-k\right)} \frac{d k}{H_{0}^{(1)}(v a)} .
\end{aligned}
$$

We are primarily interested in electromagnetic radiation in the wave zone $k_{0} R>>1, R$ is the distance from the origin of coordinates to the point of observation. Using the asymptotic representation of the Hankel function for large values of the argument $v r>1$, after passing to a spherical coordinate system $z=R \cos \vartheta, r=R \sin \vartheta(\vartheta$ is polar angle) and replacing the integration variable $k=k_{0} \cos w$ instead of the integral representation (18), we obtain

$$
\begin{gathered}
H_{\omega \varphi}^{I I I}=-i \frac{2 Q}{c t_{b} a} T_{\omega} \frac{\sqrt{k_{0}\left(k_{0}-k_{l}\right)}}{2 \pi X^{-}\left(k_{l}\right)} \sqrt{\frac{2}{k_{0} R \sin \vartheta}} e^{-i \pi / 4} \times \\
\times \int_{\pi-i \infty}^{i \infty} \frac{X^{+}\left(k_{0} \cos w\right)}{H_{0}^{(1)}\left(k_{0} \sin w\right)} \sqrt{\frac{\sin w}{1-\cos w}} e^{i k_{0} R \cos (w-\vartheta)} d w .
\end{gathered}
$$

The integration contour runs along a straight line from $\pi-i \infty$ to $\pi$, then along a segment $\pi \geq w \geq 0$ and again along a straight line from 0 to $i \infty$. In the wave zone, integral (19) can be estimated by the saddle-point method. The saddle point $w=\vartheta$ lies within the real axis line segment. As a result, we obtain the following expression for the radiation field

$$
\begin{aligned}
H_{\omega \varphi}^{I I I}=-\frac{Q}{c t_{b}} T_{\omega} \frac{\sqrt{k_{0}\left(k_{0}-k_{l}\right)}}{k_{l}} & \frac{\sin \vartheta}{\left(1-\beta_{0} \cos \vartheta\right) \sqrt{1-\cos \vartheta}} \times \\
& \times \frac{X^{-}\left(k_{0} \cos \vartheta\right)}{X^{-}\left(k_{l}\right)} \frac{e^{i k_{0} R}}{R},
\end{aligned}
$$

$\beta_{0}=v_{0} / c$. The function $X^{-}(k)$ can be calculated exactly. However, in the considered quasistatic approximation $k_{0} a<<1$, we can use the asymptotic representation of this function [13]

$$
X^{-}(k)=\sqrt{\frac{1}{\left(k_{0}-k\right) a}} \frac{\sqrt{i \ln \frac{i}{v k_{0} a}}}{\ln \frac{2 i}{v a \sqrt{2 k_{0}\left(k_{0}-k\right)}}},
$$

$v \approx 1.78$ is Euler's constant. Accordingly, the expression for the magnetic field of radiation (20) takes the form

$$
H_{\omega \varphi}^{I I I}=-\frac{Q}{c t_{b}} T_{\omega} \frac{\Lambda}{\cos \vartheta} \frac{1}{\ln \frac{i}{v k_{0} a \sin \frac{\vartheta}{2}}} \times
$$

$$
\times\left(\frac{\sin \vartheta}{1-\beta_{0} \cos \vartheta}-\frac{\sin \vartheta}{1-\cos \vartheta}\right) \frac{e^{i k_{0} R}}{R},
$$

where

$$
\Lambda=\ln \frac{\sqrt{2}}{v k_{0} a \sqrt{\beta_{0}\left(1-\beta_{0}\right)}} \approx \ln \frac{2 \gamma_{0}}{v k_{0} a},
$$

$\gamma_{0}$ is a relativistic factor. The first term in formula (21) describes the transition radiation arising from the collision of an electron bunch with the end face of a semiinfinite conducting cylinder. The second term has a singularity for the angle $\vartheta=0$. The radiation field along the cylindrical conductor goes to infinity. This effect is explained by the guiding properties of a perfectly conducting cylinder [13]. The feature of the field is integrable. The total energy flow remains finite. An analysis of the radiation pattern shows that the maximum of the radiation field in the direction characteristic of transition radiation $\vartheta=1 / \gamma_{0}$ is never formed. This is due to the strong distortion of the radiation field in this direction by radiation focused along the surface of the cylindrical conductor. Turning to infinity towards $\vartheta=0$, the amplitude of the radiation field decreases monotonically with increasing polar angle $\vartheta$. Thus, a thin semi-infinite cylinder is actually an antenna of a traveling wave excited by a relativistic electron bunch.

Directly from the form of the second term in square brackets in formula (21) it follows that the antenna radiation excites a current induced on the surface of a perfectly conducting cylinder, which propagates along the cylinder at the speed of light. Moreover, this current is in antiphase with respect to the current of the electron bunch.

A single bunch excites an electromagnetic field with a continuous frequency spectrum, and the shape of the radiation pulse is determined by the Fourier integral (2), which is conveniently represented in the form

$$
H_{\varphi}^{I I I}=\int_{0}^{\infty}\left(H_{\omega \varphi}^{I I I} e^{-i \omega t}+H_{\omega \varphi}^{I I I^{*}} e^{i \omega t}\right) d \omega .
$$

Taking into account the expression (21), relation (22) takes the form

$$
H_{\varphi}^{I I I}=\frac{Q}{c t_{b} \gamma_{0}^{2}} \frac{F(\vartheta)}{R} \int_{0}^{\infty} T_{\omega} \Lambda_{\omega} \frac{L_{\omega} \cos \omega \bar{t}+\frac{\pi}{2} \sin \omega \bar{t}}{L_{\omega}^{2}+\frac{\pi^{2}}{4}} d \omega,
$$

where

$$
\begin{gathered}
F(\vartheta)=\frac{\sin \vartheta}{\left(1-\beta_{0} \cos \vartheta\right)(1-\cos \vartheta)}, \Lambda_{\omega}=\ln \frac{\omega_{0}}{\omega}, \\
L_{\omega}=\ln \left(\frac{\omega_{0}}{\omega} \frac{1}{\gamma_{0} 2 \sin \frac{\vartheta}{2}}\right), \omega_{0}=\frac{2 \gamma_{0} c}{v a}, \bar{t}=t-\frac{R}{c} .
\end{gathered}
$$

For small polar angles $\vartheta<1$ under the condition

$$
L_{\omega}=\ln \frac{\omega_{0}}{\omega \gamma_{0} \vartheta} \sim \ln \frac{\omega_{0} t_{b}}{\gamma_{0} \vartheta}>>\frac{\pi}{4}
$$

expression (23) is essentially simplified and takes the form

$$
H_{\varphi}^{I I I}=\frac{Q}{c t_{b} \gamma_{0}^{2}} \frac{F(\vartheta)}{R} \Pi(t-R / c) .
$$




$$
\Pi(t-R / c)=\int_{0}^{\infty} T_{\omega} \frac{\ln \frac{\omega_{0}}{\omega}}{\ln \frac{\omega_{0}}{\omega \gamma_{0} \vartheta}} \cos \omega(t-R / c) d \omega
$$

describes the shape of the transition radiation pulse. For not too small angles

$$
\ln \frac{\omega_{0}}{\omega} \sim \ln \omega_{0} t_{b}>>\ln \frac{1}{\gamma_{0} \vartheta} \quad \text { or } \quad \vartheta>>\frac{1}{\gamma_{0} \omega_{0} t_{b}}
$$

we have

$$
\Pi(t-R / c)=T\left(\frac{t-R / c}{t_{b}}\right),
$$

that is, the electromagnetic pulse exactly repeats the shape of the electron bunch. Otherwise, the shape of the electromagnetic pulse will depend, albeit weakly, on the polar angle $\vartheta$ and will slightly differ from the shape of the electron bunch.

\section{CONCLUSIONS}

Transition radiation of a relativistic electron bunch, which arises when it collides with the end face of a semi-infinite perfectly conductive cylinder, is considered. The electron bunch moves along the cylinder axis. Expressions for the intensity of the magnetic field of radiation in the wave zone are obtained. It is shown that this expression contains two terms. The first term describes the actual transition electromagnetic radiation of the electron bunch. Its characteristics substantially depend on the energy (relativistic factor) of the electron bunch. The second term describes the radiation of the current induced on the surface of a perfectly conducting cylinder and propagating along the cylinder at the speed of light in vacuum. The strength of this field has integrable singularity (turns to infinity) strictly along the surface of the cylinder. The peculiarity is due to the guiding properties of a perfectly conducting cylinder. The spatial structure (directional pattern) of this electromagnetic radiation does not depend on the energy of the bunch and is determined by the geometry of the cylindrical antenna under consideration. The amplitude of the radiation field decreases monotonically with increasing polar angle $\vartheta$. The maximum of the radiation field in the direction characteristic of transition radiation $\vartheta=1 / \gamma_{0}$ is never formed. This is due to the strong distortion of the radiation field in this direction by radiation propagating along the surface of the cylindrical conductor. The shape of the emitted electromagnetic pulse has been determined.

\section{REFERENCES}

1. V.L. Ginzburg, V.N. Tsytovich. Transition radiation and transition scattering. M.: "Nauka", 1984.

2. B.M. Bolotovsky, G.V. Voskresensky. Diffraction radiation // UFN. 1966, v. 88, issue 2, p. 209-251.

3. B.M. Bolotovsky, E.A. Galstyan. Diffraction and diffraction radiation // UFN. 2000, v. 170, № 8, p. 809830.

4. F.G. Bass, V.M. Yakovenko. Theory of radiation charge passing through electrically inhomogeneous media // UFN. 1965, v. 86, issue 2, p. 189-230.

5. V.A. Balakirev, G.L. Sidel'nikov. Excitation of electromagnetic pulse by relativistic electron bunch // Technical Physics. 1999, v. 44, № 10, p. 1209-1214.

6. V.A. Balakirev, I.N. Onishchenko, D.Yu. Sidorenko, G.V Sotnikov. Wide-band emission of relativistic electron bunch in semi-infinite waveguide // Zhurnal Tekhnicheskoj Fiziki. 2002, v.72, № 2, p. 88-95 (in Russian).

7. V.A. Balakirev, N.I. Gaponenko, A.M. Gorban, et al. Excitement TEM-horn antenna by impulsive relativistic electron beam // Problems of Atomic Science and Technology. Series "Plasma Physics". 2000, № 3, p. 118-119.

8. V.A. Balakirev, A.M. Gorban', A.M. Egorov, et al. Generation of a UWB signal upon excitation spiral antenna by high-current REB // Problems of Atomic Science and Technology. Series "Nuclear Physics Investigations”. 2006, № 2, p. 85-87.

9. N.I. Gaponenko, A.M. Gorban', D.V. Gorodjanin, et al. Formation of intensive electromagnetic pulses radiated by direct excitation of an isolated whip antenna by a short-pulse // REB. Plasma Physics. 2000, v. 26, № 4, p. 387-389.

10. V.A. Balakirev, I.N. Onishchenko. Transition excitation of a perfectly conducting cylinder by relativistic electron bunches // Problems of Atomic Science and Technology. Series "Plasma Electronics and New Methods of Acceleration”. 2021, № 4, p. 96-101.

11.F.D. Gakhov. Boundary value problems. M.: "Science", 1977, $640 \mathrm{p}$.

12.N.I. Muskhelishvili. Singular integral equations. M.: "Science", 1968, 512 p.

13.L.A. Weinstein. Theory of diffraction and method of factorization. M.: "Soviet radio", 1966, 431 p.

Article received 12.10.2021

\section{ПЕРЕХОДНОЕ ИЗЛУЧЕНИЕ РЕЛЯТИВИСТСКОГО ЭЛЕКТРОННОГО СГУСТКА НА ПОЛУБЕСКОНЕЧНОМ МЕТАЛЛИЧЕСКОМ ЦИЛИНДРЕ}

\section{В.А. Балакирев, И.Н. Онищенко}

Рассмотрено переходное излучение релятивистского электронного сгустка, возникающее при его столкновении с торцом полубесконечного идеально проводящего цилиндра. Электронный сгусток движется вдоль оси полубесконечного цилиндра. Получены выражения для напряженности поля электромагнитного излучения в волновой зоне. Исследовано влияние направляющих свойств идеально проводящего цилиндра на диаграмму направленности переходного излучения.

\section{ПЕРЕХІДНЕ ВИПРОМІНЮВАННЯ РЕЛЯТИВІСТСЬКОГО ЕЛЕКТРОННОГО ЗГУСТКА НА НАПІВНЕСКІНЧЕННОМУ МЕТАЛЕВОМУ ЦИЛІНДРІ}

\section{В.А. Балакірев, І.М. Оніщенко}

Розглянуто перехідне випромінювання релятивістського електронного згустка, що виникає при його зіткненні 3 торцем напівнескінченного ідеально провідного циліндра. Електронний згусток рухається вздовж осі циліндра. Отримано вирази для напруженості поля електромагнітного випромінювання в хвильовій зоні. Досліджено вплив направляючих властивостей ідеально провідного циліндра на діаграму направленості перехідного випромінювання. 